\title{
The Efficacy of Frontal and Temporal Lobes in Detecting 2D\&3D Video Transition Using EEG
}

\author{
Negin MANSHOURI ${ }^{1}$, (D) Mesut MELEK (Masoud MALEKI) ${ }^{2}$, (D)Temel KAYIKCIOGLU ${ }^{3}$ \\ ${ }^{1}$ Department of Electrical and Electronics Engineering, Karadeniz Technical University, Trabzon, Turkey; \\ negin.manshouri@ktu.edu.tr; https://orcid.org/0000-0001-5297-5545; 05535791640 \\ 2 Department of Electronics and Automation, Gumushane University, Gumushane, Turkey; \\ mesutmelek@gumushane.edu.tr; https://orcid.org/0000-0002-7152-7788 \\ ${ }^{3}$ Department of Electrical and Electronics Engineering, Karadeniz Technical University, Trabzon, Turkey; \\ tkayikci@ktu.edu.tr, https://orcid.org/0000-0002-6787-2415
}

Received 18 June 2020; Revised 20 July 2020; Accepted 28 July 2020; Published online 28 August 2020

\begin{abstract}
Due to the three-dimensional (3D) structure of the human eye, 3D technology was used in this research. Transition to $2 \mathrm{D}$ and $3 \mathrm{D}$ analysis is important, claiming that binocular vision will lose dimension during fatigue. Thus, a single-stream video consisting of random 2D\&3D parts was watched by nine volunteers. The dynamic behavior and power spectral density (PSD) of the volunteers' brain signals were considered for a comprehensive quantitative analysis. The dominant EEG bands and time intervals were selected in 2D to 3D (2D_3D) and 3D to 2D (3D_2D) transitions by analyzing power differences based on short-time Fourier transformation (STFT). Taking into account this information, applying effective feature extraction and classification techniques, the behavioral patterns of channels representing the brain lobes of the volunteers were analyzed. Hjorth parameters and statistical methods were used as feature extraction methods. The k-nearest neighbors (k-NN) and linear discriminant analysis (LDA) algorithms were applied to classify 2D_3D and 3D_2D transitions. The results revealed that, thanks to the comprehensive classification analysis of the 2D_3D and 3D_2D transitions, the change in the activity power of the brain cortex can be represented. The dominance of the temporal and frontal lobes between the channels representing these transitions cannot be excluded.
\end{abstract}

Keywords: EEG, 2D\&3D, transition, classification, anaglyph

\section{EEG Kullanarak 2D\&3D Video Geçişinin Tespitinde Frontal ve Temporal Lobların Etkinliği}

Öz

İnsan gözünün üç boyutlu (3B) yapısı nedeniyle, bu araştırmada 3B teknolojisi kullanılmıştır. Binoküler görmenin yorgunluk esnasında boyut kaybedeceğini iddia ederek $2 \mathrm{~B}$ ve 3B'ye geçiş analizi önemlidir. Böylece rastgele 2B ve 3B parçalardan oluşan tek akışlı bir video dokuz gönüllü tarafından izlenilmiştir. Gönüllülerin beyin sinyallerinin dinamik davranışı ve güç spektral yoğunluğu (GSY), kapsamlı bir nicel analiz için dikkate alınmıştır. Baskın EEG bantları ve zaman aralıkları, kısa zamanlı Fourier dönüşümüne (KZFD) dayalı güç farklılıklarını analiz ederek 2B'den 3B'ye (2B_3B) ve 3B'den 2B'ye (3B_2B) geçişlerde seçilmiştir. Bu bilgiler dikkate alınarak, etkili özellik çıkarımı ve sınıflandırma teknikleri uygulanarak, gönüllülerin beyin loblarını temsil eden kanalların davranıș kalıpları analiz edilmiștir. Öznitelik çıkarma yöntemleri olarak Hjorth parametreleri ve istatistiksel yöntemler kullanılmıştır. 2B_3B ve 3B_2B geçişlerini sınıflandırmak için en yakın komşular (e-YK) ve doğrusal diskriminant analiz (DDA) algoritmaları uygulanmıştır. Sonuçlar, 2B_3B ve 3B_2B geçişlerinin kapsamlı sınıflandırma analizi sayesinde beyin korteksinin aktivite gücünün değiş̧iminin temsil edilebileceğini ortaya koymuştur. Bu geçişleri temsil eden kanallar arasındaki temporal ve frontal lobların baskınlığı göz ardı edilemez.

Anahtar Kelimeler: EEG, 2B\&3B, geçiş, sinıflandırma, anaglif 


\section{Introduction}

Studies in 2D\&3D areas have been rapidly developing because these areas form real feelings and a sense of sensibility in people. This development is manifested in different domains of the scientific world, from the medical to the entertainment and education sectors. These areas of application have been taken into account by researchers in the category of entertainment [1], health [2]-[3], industry, and architecture [4]. Thanks to the excitement created by this technology, it attracts a great deal of attention from young people [5]. In addition to being the focus of attention of 3D technology, it has some disadvantages. In case of frequent use, it can lead to habits as well as health problems such as headaches, eye fatigue, and physical discomfort [6]. 3D technology has a long history, spanning from the invention of stereoscope in the 18th century to the 3D TV in the 20th century [7]-[8].

Some studies have evaluated the visual differences resulting from watching 2D\&3D images using the electroencephalogram (EEG) [9]-[10]. Measurement of ionic voltage fluctuations of brain neurons is defined as EEG. The EEG method is preferred because it is the most important and reliable physiological measurement in evaluating mental activities. This method is the physiological method used to record the electrical activity produced by the brain through electrodes placed on the scalp surface. By using the frequency region of signals, especially frequency bandwidths, EEG enables this information to help reveal the functional states of the brain [11]-[12].

In order to grasp the logic of 3D technology, the human eye structure must be taken into account. It is also useful to learn how the eyes and the brain work. People see the world with eyes looking in two directions at a distance of about $6 \mathrm{~cm}$ [13]. Unlike some animals [14], people have the ability to see binoculars due to the eye structures. Each eye has a certain field of view when the other eye is closed. Although the visual information in the environment can only be perceived with one eye, the fields of vision of the eyes overlap. This information is transmitted to a region of the brain behind the head, and synthesizes overlapping visual information to create a single coordinated image. In summary, what we see is the result of signals sent from the eyes to the brain. In general, the brain receives signals from both eyes (ocular) at the same time. The information contained in the signal for each eye is slightly different, and with a well-functioning binocular vision, the brain can understand these differences; Also it can be used to assess distances and coordinate eye movements [15]-[16]. This difference in the retina is known as binocular disparity. In 1838, Wheatstone showed that these subtle differences between retinal images are detected by the brain and provide a powerful clue to the depth structure of the world around us [16]. This ability is known as (binocular) stereoscopic or stereo vision. It is also often described as a 3D vision. One of the reasons why binocular vision is so important is that it allows to perceive depth and relationships between objects.

EEG analysis of 2D and 3D technology can generally be classified as eye fatigue detection [17], game analysis [18], [19], detection of power spectrum differences in brain waves [20], [8], and investigation of the effects of stereoscopic disparity on event-related potentials [21]. Few of these studies have focused on a brief look analysis of brain signals in 2D and 3D video watching instead of comprehensive analysis, and none of them has examined the 2D and 3D transitions of video watching modes.

Taking into account the basis of 2D and 3D technology, until 2011 very few studies in this area actually focus on the quantitative benefits of 3D visualization [22]. Many studies have proved that despite the complexity of 3D technology, it performs better than 2D in terms of model visualization [23]. Thanks to the excitement and realistic feelings created by 3D technology, its expansion continues rapidly in today's research. Therefore, it is important to understand the comprehensive analysis of brain activation patterns underlying 2D and 3D studies [24].

The viewing of the television screen from different distances and how this distance affects the human visual system was also evaluated in detail [17]. Time interval and screen distance are among the important factors in 3D watching. The visual fatigue survey for 2D versus 3D has been carried out in [25]. By using EEG-based 2D and 3D concentrated games, a biomedical feedback system has been developed in [3]. In this study, by utilizing 2D and 3D technology and nonlinear methods concentration check on human individuals was performed . In five physiological status analysis of [18], EEG signal complexity was evaluated using two parameters. In the case of openness of the eye and in 3D mode, 
complexity increasing was observed. The depth degree of 3D images and the relationship between visual disturbance were also explored in detail in [21]. As a result of the depth of 3D technology, a considerable increase in ERP components (P1) was observed compared to 2D. The amplitude of this component can be adjusted by changing the degree or perception of depth. Brain areas sensitive to binocular vision and perception of depth were analyzed in [9], [26] based on Brodmann areas (BA). These regions were determined as temporal, parietal and occipital.

This study is a basic step towards evaluating our hypothesis which is based on the human eye structure. In this hypothesis, the analysis of the moment of transition to 2D_3D and 3D_2D is important, claiming that 3D vision will lose dimension during fatigue. Behavior analysis and classification of brain signals in these transitions are the main goals of this research. In the process of watching a single anaglyph video consisting of random 2D and 3D parts, electrophysiological responses were discussed in detail. In a brief description of this detailed analysis, the power spectrograms of the electrophysiological responses achieved on the presentation of the 2D_3D and 3D_2D transitions were obtained using the STFT. Thus, it is possible to get an idea about the behavior of each channel by considering the power spectrum difference of transition states. In addition, based on this visual information, important frequency bands, time windows, and channels that maximize the power difference of the transitions were brought to the fore. In order to obtain an acceptable performance classification technique, effective feature extraction methods were applied by selecting dominant frequency and time intervals.

By evaluating this hypothesis, the effects of 2D_3D and 3D_2D transitions on brain signals in terms of time, frequency, and channel can be determined in a single video of 2D and 3D parts. In this way, it is hoped that the researchers will be able to provide information about the parameters to be considered in the studies on 2D and 3D technology (visual processing, classification, early detection of drivers falling asleep, etc.). The results of the study will contribute to the literature on the comprehensive brain dynamics underlying 2D and 3D technology and the parameters affecting these dynamics.

\section{Material and Method}

In this section, information was given about volunteers, video production, EEG recording, data processing, and analysis methods.

\subsection{Volunteers}

Nine volunteers (four women and five men) without neurophysiological disorders participated in the experiments. The average age and standard deviation (SD) were 34.12 and 2.072 years, respectively. The experiments were carried out according to the experimental procedures No. 24237859-806 approved by the Ethics Committee of the Faculty of Medicine, Karadeniz Technical University. Volunteers were informed about this protocol in advance, and their written consent was obtained.

\subsection{Video Production}

A 135-second video was watched by the volunteers five times. This video consisted of 8-second 2D and 3D random parts. In the adjustment of this anaglyph video, the 3D version of the SAW video [27] was converted to 2D using the Xilisoft 3D Video Converter program [28]. Later, the 3D version was converted to a 3D anaglyph format using the IQmango 3D converter program [29]. The resulting parts were combined by applying the Idoo video program [30]. The selected glasses must be independent of any imaging system to view 2D and 3D video segments in a single video, considering TV features [20]. Thus, anaglyph glasses [31] were found to be suitable for this scenario according to the working principle.

\subsection{EEG Recording}

A 32-channel Micromed Italy Brian Quick device was used for EEG data recording. The experimental environment and the system are presented in Figure 1. The EEG cap consisted of 21 electrodes, and the 
animation graphic of this cap is presented in Figure 2. In the experiments, the $\mathrm{Cz}$ electrode was determined as a ground electrode. EEG recordings were made by trying to keep the electrode resistance values below $10 \mathrm{~K}$ Ohms on average. Considering the EEG bandwidth of 0-100 Hz, sampling frequency (fs) was chosen as $512 \mathrm{~Hz}$ to ensure sufficient time resolution and prevent data exchange in long-term recordings.

The experiments were conducted in a dimly lit room. The subjects were asked to sit in a comfortable chair about $85 \mathrm{~cm}$ from the TV stand, relax, and minimize body movements during the experiment. Each recording lasted 135 seconds and was repeated five times. There was insulation isolation in the EEG system used to protect each experiment from the electrical impact. In the device used, a filter that passes between $0.1-120 \mathrm{~Hz}$ was applied to clear the recorded EEG signals from internal and external artifacts. In addition, a $50 \mathrm{~Hz}$ notch filter was employed to eliminate mains noise.

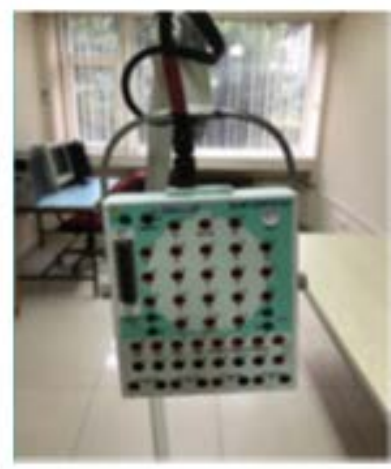

(a)

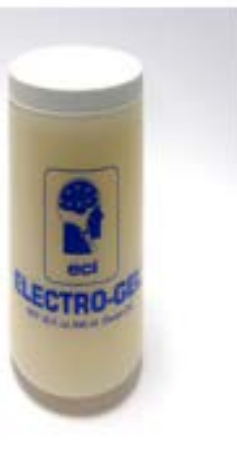

(b)

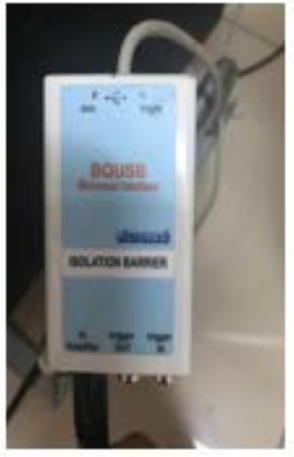

(c)

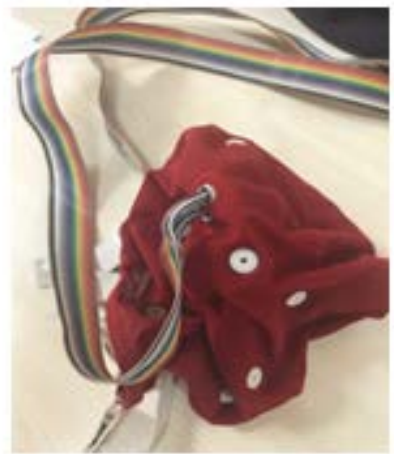

(d)

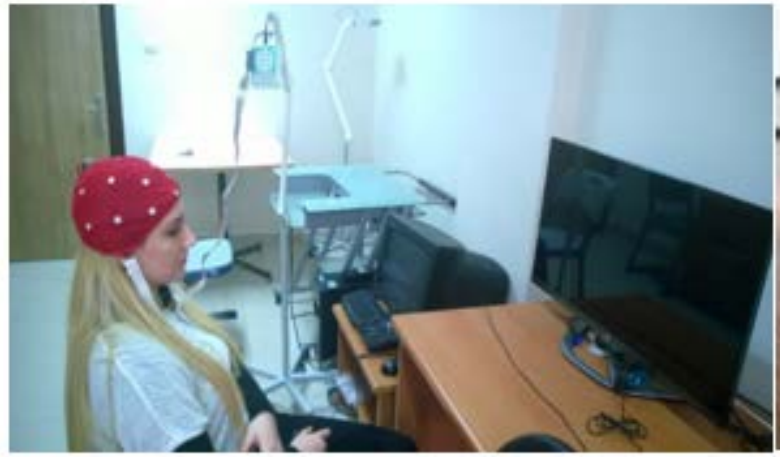

(e)

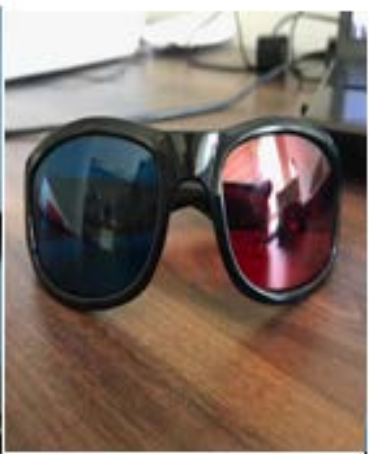

(f)

Figure 1 EEG system a) EEG amplifier, (b) gel, (c) EEG isolation, (d) EEG cap, (e) EEG recording area, (f) Anaglyph glasses

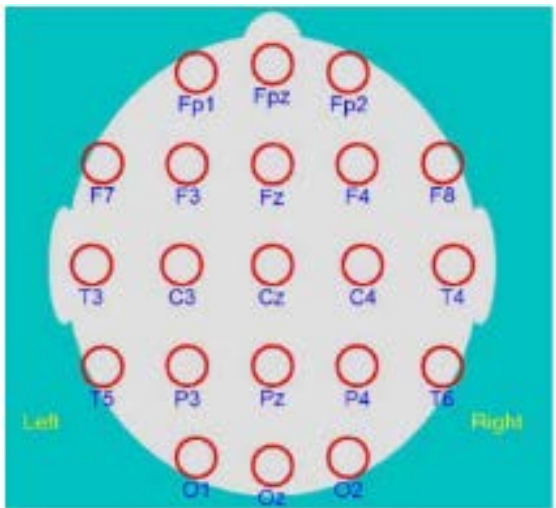

Figure 2 The animation graphic of the EEG cap 


\subsection{Scenario}

In this scenario, the movie consisting of random 2D and 3D videos was watched by the volunteers. The aim was to analyze and classify 2D and 3D transitions. An EEG recording example of the sequence of the video, consisting of 8-second 2D and 3D random parts, is illustrated in Figure 3. In Figure 3, the orange-colored arrows represent the 2D_3D transitions, and the green arrows the 3D_2D transitions. In the transition analysis, there is a total of nine transitions (five 2D_3D and four 3D_2D transitions). The window length of five seconds was taken into account in terms of epoch category. This category is presented in Table 1. In this table, according to Figure 3, window lengths of 5 seconds were selected for analysis, considering the critical transitions from 2D to 3D (2D_3D) and from 3D to 2D (3D_2D). As mentioned before, each recording was repeated five times. Thus, due to the window length of 5 seconds and the existence of nine epochs, a total of 45 epochs was included in the analysis.

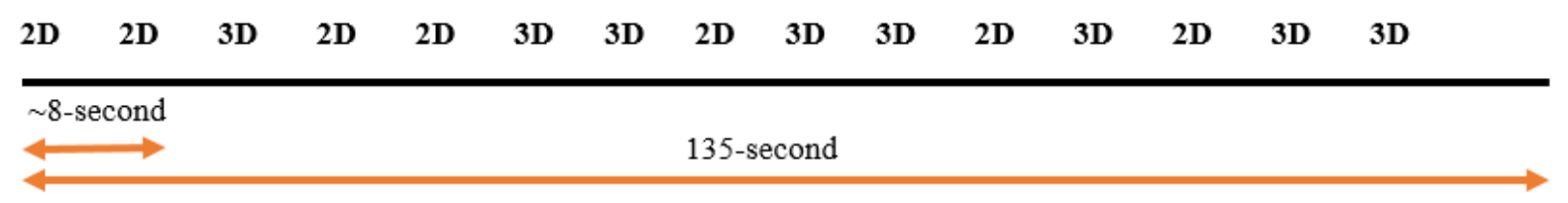

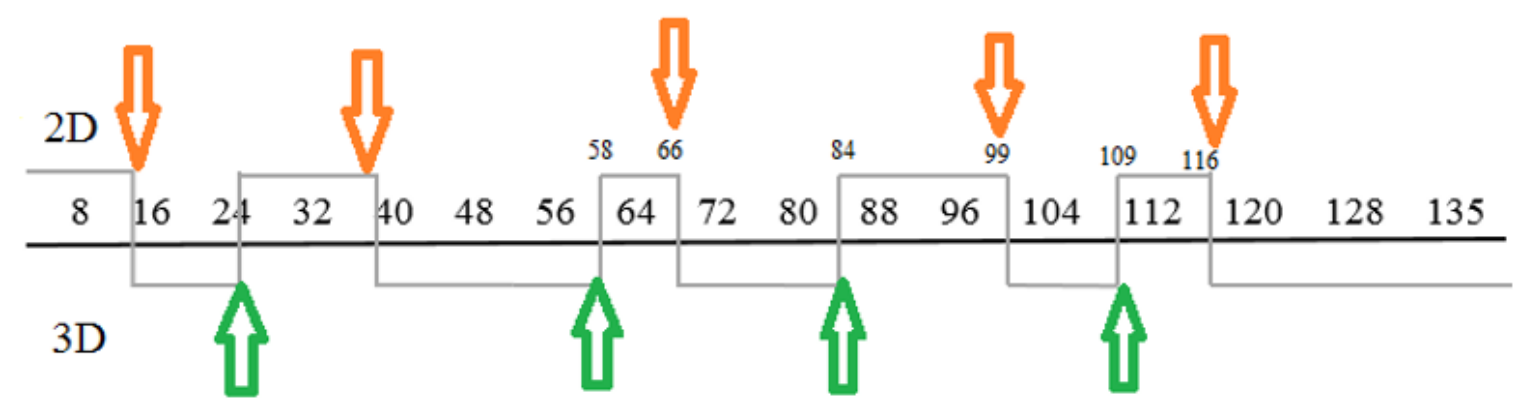

Figure 3. Details of the video

Table 1. Epoch category

\begin{tabular}{|l|l|}
\hline 5-second window length from 2D_3D & 5-second window length from 3D_2D \\
\hline $13-18,37-42,63-68,96-101,113-118$ & 21-26, 55-60, 81-86, 106-111 \\
\hline
\end{tabular}

\subsection{Preprocessing}

Following the EEG signal recording, the recordings were split into two sub-data sets, namely 5-second window lengths covering 2D_3D and 3D_2D transitions. Since there were nine transitions in each of the five trials, 45 epochs were taken into account. In addition, each sub-data set represents 20 channels of nine volunteers. To take the first step of data analysis, the average of five trials was calculated in order to get an idea of the choice of dominant bands and to minimize noise. In addition to applying a notch filter at $50 \mathrm{~Hz}$ to clear the network noise [32], a third-order bandpass Butterworth filter to clean the noise signal in the frequency range of $1-55 \mathrm{~Hz}$ was employed. The order of the filter was chosen by trial and error. All the data had to be normalized in order to eliminate this problem and provide the opportunity to compare different volunteers. The z-score normalization method [33] was adopted to minimize amplitude changes and enhance the classification performance.

\subsection{Time-Frequency Analysis Process and Band Selection}

Time-frequency analysis was applied to get a more effective idea about the processed data. Thus, in the 2D_3D and 3D_2D transition analysis, the time intervals representing the maximum difference of PSD and the dense frequency range were taken into account. In other words, the behavior change between 2D_3D and 3D_2D transitions is well demonstrated. The spectrogram method based on STFT [34] was used to accomplish this. The STFT is an expanded version of the traditional Fourier analysis used for the analysis of non-stationary data. This technique analyzes the local frequency content of the signal 
with floating windowing [35]. By adjusting the width of this window, valuable information about unpredictable EEG signals can be obtained. Due to the unknown nature of the EEG signals, the softbehavior Hanning window was chosen. One of the suitable features of this window is that it has a wide peak unlike its narrow side lobes. Thus, in order to capture the related time and frequency resolution, taking into account the frequency range desired, the window size was considered as 512 sample lengths. The overlapping of the window was noted as 'window size -1 ' [8].

The transition effect of 2D_3D and 3D_2D anaglyph video watching was evaluated on the power of brain signals for different frequency bands. To gain the goal, the average EEG power spectrogram of all the epochs belonging to the nine volunteers in each sub-data set was achieved separately for 20 channels. As a result, when the average EEG power spectrograms were investigated for the effective time windows, differences were observed between the average power spectrograms of the 2D_3D and 3D_2D transitions on the dominate frequency and channels. Based on the spectrogram graph, in 2D_3D and 3D_2D transition analysis, the delta band was determined as the dominant band in approximately all EEG channels representing brain lobes during the comparison phase. Considering the epoch averages, this band selection was calculated according to the average results of the volunteers in each channel. In the next step, taking into account the dominant band and time intervals obtained from this section, the feature extraction process was performed for 5-second epochs by selecting 1-1.5 and 1.5-3-second time intervals at 2,3 , and $4 \mathrm{~Hz}$ frequencies.

\subsection{Feature Extraction}

The main purpose of the feature extraction method in EEG signals is the extraction of features that effectively reflect the frequency, time, and spatial properties of the signal. Here, selecting effective values of time-frequency windows that best represent the differences between the power spectra showing the 2D_3D and 3D_2D transitions can be defined as the feature extraction method. Considering the STFT-based spectrogram graph, two feature extraction methods were utilized in the dominant band and time intervals. One is the SD and max of statistical functions, and the other is Hjorth parameters [36]. Three parameters of the Hjorth method are activity, mobility, and complexity. The mathematical representation of these parameters is shown respectively in Equations 1, 2, and 3.

$$
\begin{gathered}
\text { Activity }=\frac{1}{N} \sum_{n=0}^{N-1}[x(n)-\bar{x}]^{2}=\operatorname{variance}(x(n)) \\
\text { mobility }=\sqrt{\frac{\operatorname{Activity}(\dot{x}(n))}{\operatorname{Activity}}}=\sqrt{\frac{\operatorname{variance}(\dot{x}(n))}{\operatorname{variance}(x(n))}} \\
\text { Complexity }=\sqrt{\frac{\text { variance }(\dot{x}(n)) \operatorname{variance}(x(n))}{\operatorname{variance}(\dot{x}(n))^{2}}}
\end{gathered}
$$

where $\dot{x}(n)$ and $\dot{x}(n)$ are the first and second derivatives of the signal, respectively. While these three parameters contain information on the frequency spectrum of a signal, they also help analyze the signals in the time domain. In addition, lower computational complexity can be achieved by their use.

Ultimately for each channel, in the SD and max feature extraction method, considering the dominant frequency and time windows, the feature vector dimension was determined as $3 * 2 * 2$ and in the Hjorth method as $3 * 3$. The band selection and feature extraction method is summarized in Figure 4 as block diagram representation. 


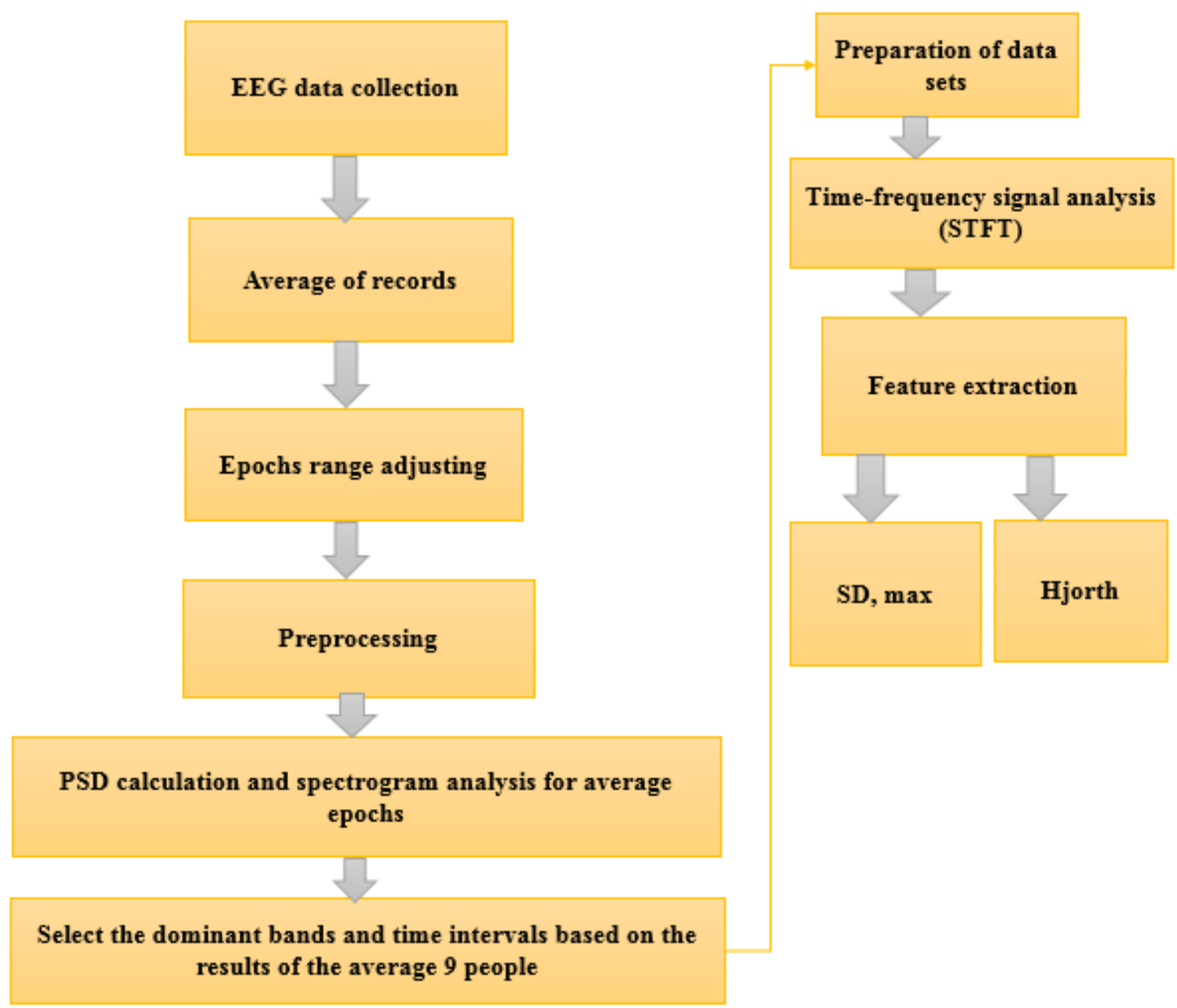

Figure 4 Flow diagram summarizing band selection and feature extraction method

\subsection{Classification Techniques and Performance Evaluation of Classification}

To achieve the goals of this study, namely 2D_3D and 3D_2D transition classification, k-NN and LDA classification techniques were employed. Since this study has two classes, the chance level is 50\%. Additionally, sensitivity and specificity [16] were used to evaluate classifier performance and classification accuracy. The basic definition of these techniques is briefly presented below.

The supervised and simple k-NN technique that is sensitive to the local structure of the data is also used in pattern recognition for different classification and regression processes [27]. This algorithm has a lazy learning mechanism, and it tries to make estimations by memorizing the training dataset instead of learning it. During the estimation process, it looks for the nearest neighbors, taking into account the entire data set [28]. The class of the new sample is determined by looking at the closest k neighbor's class according to the distance in the learning set. In this study, these distances were calculated using the Euclidean distance function. Setting the $\mathrm{k}$ or neighborhood parameter directly affects the performance of this algorithm. If $\mathrm{k}$ is too small, it affects noise sensitivity, and it is too large, it affects the neighbors of other classes. There is no clear method for choosing $\mathrm{k}$. As used in this study, in general, the optimum $\mathrm{k}$ is selected by using the cross-validation method.

The LDA algorithm is used in machine learning and pattern recognition to separate two or more classes of problems. Its basic principle is based on the linear combination of features. This algorithm actually reveals the modeling differences of classes by moving the high-dimensional space features to a smaller size [29].

To evaluate the classification result validation, K-fold cross-validation was applied. K-fold crossvalidation was employed to evaluate the classification result validation. In this study, the $\mathrm{K}$ value was chosen as 10. After cross-validation, the $\mathrm{k}$ value of $\mathrm{k}-\mathrm{NN}$ was obtained. Here, class 2D_3D and 3D_2D 
were defined as the positive and negative sample, respectively. To move on to the classification stage, the epochs of each class were randomly divided into two test and training sets. The classification process was repeated 20 times to avoid unlucky splitting.

\section{The Research Findings and Discussion}

If we want to summarize the outcome of the time-frequency analysis of EEG data, the difference of the average power spectra based on the average epochs in the evaluation of 2D_3D and 3D_2D transitions was shown for 20 different channels. Thus, dominant time intervals and effective frequency band were determined in this transition classification. As a result, the delta and 1-1.5 and 1.5-3 seconds time intervals were the best criteria representing the power difference. The k-NN classification results of 9 volunteers (V) in each channel were shown in Tables 2 and 3 for SD, max and Hjorth feature extraction methods, respectively. Likewise, LDA classification results were presented in Tables 4 and 5, respectively.

When looking at the classification results of 9 volunteers on 20 channels, it is not overlooked that the LDA and Hjorth methods are generally more successful. With a general view in terms of brain lobes, the frontal, temporal and partially parietal lobes appear to be highly successful. Considering the general average classification result in the LDA classification algorithm, T3 and Pz were chosen as the channels with the best results in SD, max and Hjorth methods with success of $66.67 \%$ and $66.25 \%$, respectively. Since this study consists of two classes, it is observed that the classification results are low. In this case, it is necessary to increase these results by applying another method.

Table 2 k-NN classification results for nine volunteers in each channel, and the average classification accuracy (SD, max)

\begin{tabular}{|c|c|c|c|c|c|c|c|c|c|c|}
\hline & V1 & V2 & V3 & V4 & V5 & V6 & V7 & V8 & V9 & Average \\
\hline Fp1 & 60 & 55 & 50 & 65 & 55 & 60 & 60 & 60 & 60 & 58.33 \\
\hline Fpz & 50 & 50 & 55 & 60 & 60 & 70 & 65 & 65 & 45 & 57.78 \\
\hline Fp2 & 45 & 60 & 55 & 65 & 65 & 60 & 70 & 65 & 50 & 59.45 \\
\hline F3 & 50 & 65 & 55 & 50 & 65 & 50 & 55 & 65 & 70 & 58.33 \\
\hline F4 & 65 & 50 & 50 & 65 & 55 & 65 & 55 & 60 & 55 & 57.78 \\
\hline F7 & 55 & 50 & 55 & 60 & 45 & 65 & 50 & 60 & 55 & 55 \\
\hline F8 & 45 & 30 & 65 & 55 & 65 & 65 & 60 & 45 & 35 & 51.67 \\
\hline C4 & 65 & 45 & 60 & 55 & 65 & 50 & 45 & 45 & 60 & 54.45 \\
\hline C3 & 75 & 60 & 50 & 50 & 60 & 55 & 85 & 35 & 55 & 58.33 \\
\hline Fz & 50 & 50 & 65 & 45 & 85 & 50 & 65 & 60 & 60 & 58.88 \\
\hline P3 & 65 & 55 & 60 & 60 & 70 & 70 & 55 & 55 & 70 & 62.23 \\
\hline P4 & 50 & 60 & 65 & 60 & 40 & 70 & 55 & 55 & 65 & 57.78 \\
\hline Pz & 60 & 50 & 55 & 70 & 70 & 55 & 70 & 60 & 75 & 62.78 \\
\hline O1 & 50 & 60 & 55 & 50 & 60 & 45 & 55 & 55 & 60 & 54.45 \\
\hline O2 & 60 & 50 & 60 & 50 & 50 & 75 & 55 & 55 & 65 & 57.78 \\
\hline T3 & 60 & 60 & 45 & 45 & 45 & 55 & 50 & 60 & 60 & 53.33 \\
\hline T4 & 55 & 55 & 50 & 75 & 50 & 55 & 50 & 50 & 75 & 57.22 \\
\hline T5 & 60 & 55 & 40 & 55 & 50 & 60 & 65 & 50 & 50 & 53.12 \\
\hline T6 & 65 & 55 & 55 & 65 & 55 & 60 & 50 & 60 & 60 & 58.33 \\
\hline Oz & 60 & 65 & 45 & 65 & 60 & 45 & 55 & 65 & 65 & 58.33 \\
\hline
\end{tabular}


Manshouri et. al

Table 3. The k-NN classification results of nine volunteers in each channel, and the average classification accuracy (Hjorth)

\begin{tabular}{|c|c|c|c|c|c|c|c|c|c|c|}
\hline & V1 & V2 & V3 & V4 & V5 & V6 & V7 & V8 & V9 & Average \\
\hline Fp1 & 60 & 60 & 55 & 65 & 55 & 60 & 65 & 60 & 60 & 60 \\
\hline Fpz & 55 & 70 & 50 & 60 & 60 & 70 & 65 & 65 & 45 & 60 \\
\hline Fp2 & 40 & 65 & 50 & 65 & 60 & 60 & 70 & 65 & 50 & 58.34 \\
\hline F3 & 65 & 55 & 55 & 60 & 65 & 50 & 55 & 65 & 75 & 60.55 \\
\hline F4 & 55 & 65 & 60 & 65 & 60 & 65 & 55 & 60 & 55 & 60 \\
\hline F7 & 35 & 70 & 65 & 60 & 40 & 50 & 40 & 60 & 50 & 52.22 \\
\hline F8 & 60 & 60 & 50 & 55 & 75 & 65 & 60 & 40 & 35 & 55.56 \\
\hline C4 & 35 & 50 & 55 & 55 & 65 & 50 & 45 & 45 & 60 & 51.12 \\
\hline C3 & 60 & 55 & 70 & 55 & 60 & 55 & 80 & 25 & 50 & 56.67 \\
\hline Fz & 50 & 50 & 50 & 45 & 75 & 60 & 65 & 60 & 50 & 56.11 \\
\hline P3 & 50 & 55 & 45 & 65 & 70 & 70 & 55 & 55 & 70 & 59.44 \\
\hline P4 & 60 & 45 & 55 & 60 & 40 & 70 & 50 & 50 & 65 & 55 \\
\hline Pz & 70 & 65 & 60 & 70 & 70 & 55 & 70 & 60 & 60 & 64.45 \\
\hline O1 & 60 & 30 & 60 & 60 & 60 & 55 & 70 & 60 & 60 & 57.22 \\
\hline O2 & 60 & 40 & 75 & 55 & 40 & 75 & 55 & 55 & 55 & 56.67 \\
\hline T3 & 50 & 65 & 60 & 45 & 45 & 55 & 50 & 60 & 60 & 54.45 \\
\hline T4 & 50 & 55 & 65 & 80 & 50 & 60 & 50 & 50 & 75 & 59.44 \\
\hline T5 & 65 & 60 & 50 & 55 & 50 & 60 & 65 & 50 & 65 & 57.78 \\
\hline T6 & 60 & 60 & 45 & 65 & 50 & 60 & 65 & 55 & 55 & 57.22 \\
\hline Oz & 70 & 50 & 55 & 65 & 60 & 55 & 60 & 65 & 65 & 60.55 \\
\hline
\end{tabular}

Table 4. LDA classification results of nine volunteers in each channel, and the average classification accuracy (SD, max)

\begin{tabular}{|c|c|c|c|c|c|c|c|c|c|c|}
\hline & V1 & V2 & V3 & V4 & V5 & V6 & V7 & V8 & V9 & Average \\
\hline Fp1 & 55 & 45 & 50 & 50 & 60 & 70 & 55 & 45 & 55 & 53.89 \\
\hline Fpz & 40 & 50 & 50 & 55 & 60 & 65 & 55 & 60 & 65 & 55.56 \\
\hline Fp2 & 40 & 50 & 55 & 70 & 65 & 60 & 45 & 65 & 55 & 56.11 \\
\hline F3 & 55 & 50 & 50 & 55 & 60 & 60 & 55 & 45 & 70 & 55.56 \\
\hline F4 & 45 & 40 & 55 & 65 & 70 & 70 & 60 & 55 & 60 & 57.78 \\
\hline F7 & 60 & 45 & 45 & 50 & 60 & 60 & 80 & 65 & 70 & 59.45 \\
\hline F8 & 45 & 65 & 50 & 55 & 40 & 55 & 65 & 50 & 60 & 53.89 \\
\hline $\mathbf{C 4}$ & 55 & 50 & 60 & 55 & 70 & 70 & 60 & 65 & 70 & 61.67 \\
\hline C3 & 75 & 55 & 50 & 65 & 55 & 55 & 80 & 75 & 70 & 64.45 \\
\hline Fz & 55 & 50 & 80 & 50 & 65 & 55 & 65 & 70 & 45 & 59.44 \\
\hline $\mathbf{P 3}$ & 65 & 75 & 70 & 65 & 70 & 50 & 60 & 60 & 60 & 63.89 \\
\hline P4 & 55 & 55 & 60 & 60 & 65 & 50 & 40 & 60 & 60 & 56.12 \\
\hline Pz & 55 & 80 & 60 & 65 & 55 & 50 & 80 & 90 & 60 & 66.11 \\
\hline $\mathbf{O 1}$ & 45 & 55 & 55 & 50 & 60 & 50 & 45 & 40 & 75 & 52.77 \\
\hline $\mathbf{O 2}$ & 50 & 70 & 55 & 50 & 55 & 50 & 45 & 70 & 60 & 56.11 \\
\hline T3 & 60 & 60 & 75 & 55 & 85 & 70 & 65 & 60 & 70 & 66.67 \\
\hline T4 & 60 & 70 & 45 & 65 & 50 & 60 & 60 & 65 & 75 & 61.12 \\
\hline T5 & 50 & 75 & 65 & 65 & 65 & 50 & 70 & 65 & 60 & 62.78 \\
\hline T6 & 65 & 60 & 50 & 60 & 55 & 55 & 35 & 55 & 60 & 55 \\
\hline Oz & 50 & 60 & 65 & 55 & 65 & 60 & 40 & 60 & 65 & 57.78 \\
\hline
\end{tabular}


Table 5. LDA classification results of nine volunteers in each channel, and the average classification accuracy (Hjorth)

\begin{tabular}{|c|c|c|c|c|c|c|c|c|c|c|}
\hline & V1 & V2 & V3 & V4 & V5 & V6 & V7 & V8 & V9 & Average \\
\hline Fp1 & 55 & 60 & 40 & 50 & 60 & 70 & 55 & 45 & 55 & 54.37 \\
\hline Fpz & 70 & 55 & 45 & 70 & 60 & 65 & 55 & 60 & 65 & 60 \\
\hline Fp2 & 70 & 55 & 45 & 55 & 65 & 60 & 45 & 65 & 55 & 57.5 \\
\hline F3 & 50 & 80 & 60 & 65 & 60 & 60 & 55 & 45 & 70 & 59.37 \\
\hline F4 & 45 & 45 & 55 & 50 & 70 & 70 & 60 & 55 & 60 & 56.25 \\
\hline F7 & 70 & 70 & 50 & 55 & 60 & 60 & 80 & 65 & 70 & 63.75 \\
\hline F8 & 85 & 60 & 60 & 55 & 40 & 55 & 65 & 50 & 60 & 58.75 \\
\hline $\mathbf{C 4}$ & 65 & 55 & 45 & 65 & 70 & 70 & 60 & 65 & 70 & 61.87 \\
\hline $\mathbf{C 3}$ & 60 & 60 & 55 & 50 & 55 & 55 & 80 & 75 & 70 & 61.25 \\
\hline Fz & 75 & 60 & 45 & 65 & 65 & 55 & 65 & 70 & 45 & 62.5 \\
\hline $\mathbf{P 3}$ & 65 & 65 & 55 & 60 & 70 & 50 & 60 & 60 & 60 & 60.62 \\
\hline $\mathbf{P 4}$ & 50 & 65 & 65 & 65 & 65 & 50 & 40 & 60 & 60 & 57.5 \\
\hline Pz & 70 & 65 & 70 & 50 & 55 & 50 & 80 & 90 & 60 & 66.25 \\
\hline $\mathbf{O 1}$ & 55 & 65 & 60 & 50 & 60 & 50 & 45 & 40 & 75 & 53.12 \\
\hline $\mathbf{O 2}$ & 55 & 75 & 45 & 55 & 55 & 50 & 45 & 70 & 60 & 56.25 \\
\hline T3 & 70 & 45 & 50 & 65 & 85 & 70 & 65 & 60 & 70 & 63.75 \\
\hline T4 & 65 & 60 & 65 & 65 & 50 & 60 & 60 & 65 & 75 & 61.25 \\
\hline T5 & 50 & 50 & 65 & 60 & 65 & 50 & 70 & 65 & 60 & 59.37 \\
\hline T6 & 65 & 65 & 65 & 55 & 55 & 55 & 35 & 55 & 60 & 56.25 \\
\hline $\mathbf{O z}$ & 75 & 65 & 60 & 55 & 65 & 60 & 40 & 60 & 65 & 60 \\
\hline
\end{tabular}

\subsection{Temporal and Frontal Lobe Activity Using Voting}

The three channels with the best accuracy rates in all volunteers for all classifiers are listed in Table 6 below. Considering the table of the results presented in the previous section, it can be seen that the channels in the temporal and frontal regions are effective and dominant. Therefore, we can say that these regions are important in 2D and 3D video watching. In order to improve the classification results, three channels from the frontal and temporal lobes with the best accuracy in each volunteer are identified in Table 7. That is, in Table 6, the channels from the parietal, occipital, and central lobes are replaced by the best channels from the frontal and temporal lobes. For a good understanding, for example, in the first volunteer (V1) of Table 2, in the three best channels (C3, F4, T6), the C3 channel was replaced by the channel (Fp1), which gave the best result from the frontal or temporal lobe. The combination of these channels is presented in Table 7.

Table 6 Three channels with the best success in nine volunteers for SD, max, and Hjorth feature extraction methods

\begin{tabular}{|c|c|c|c|c|}
\hline \multirow{2}{*}{} & \multicolumn{2}{|c|}{ k-NN } & \multicolumn{2}{c|}{ LDA } \\
\cline { 2 - 5 } & SD and max & Hjorth & SD and max & Hjorth \\
\hline V1 & C3, F4, T6 & Oz, Pz, F3 & C3, T6, P3 & F8, Fz, Oz \\
\hline V2 & F3, Oz, T3 & Fpz, F7, Fp2 & Pz, T5, P3 & F3, O2, F7 \\
\hline V3 & F8, Fz, P4 & O2, C3, T4 & Fz, T3, P3 & Pz, T5, T4 \\
\hline V4 & T4, Pz, Fp1 & T4, Pz, Fp1 & Fp2, T4, T5 & Fp2, F4, T4 \\
\hline V5 & Fz, P3, Pz & F8, Fz, P3 & T3, F4, P3 & T3, F4, C4 \\
\hline V6 & O2, Fpz, P4 & O2, Fpz, P3 & Fp1, F4, T3 & Fp1, F4, T3 \\
\hline V7 & C3, Fp2, Pz & C3, Fp2, Pz & F7, Pz, C3 & F7, C3, Pz \\
\hline V8 & Fpz, Fp2, F3 & Fpz, Fp2, F3 & Pz, C3, O2 & Pz, C3, Fz \\
\hline V9 & T4, Pz, F3 & T4, F3, P3 & T4, F8, P3 & T4, O1, F3 \\
\hline
\end{tabular}


Table 7 The three best channels selected from the frontal and temporal lobes

\begin{tabular}{|c|c|c|c|c|}
\hline \multirow{2}{*}{} & \multicolumn{2}{|c|}{ k-NN } & \multicolumn{2}{c|}{ LDA } \\
\cline { 2 - 5 } & SD and max & Hjorth & SD and max & Hjorth \\
\hline V1 & F4, T6, Fp1 & F3, T5, Fp1 & T6, F7, T4 & F8, Fz, Fpz \\
\hline V2 & F3, T3, Fp2 & Fpz, F7, Fp2 & T5, T4, F8 & F3, F7, T6 \\
\hline V3 & F8, Fz, T6 & T4, F7, F4 & Fz, T3, T5 & T5, T4, T6 \\
\hline V4 & T4, Fp1, T6 & T4, Fp1, Fp2 & Fp2, T4, T5 & Fp2, F4, T4 \\
\hline V5 & Fz, Fp2, F3 & F8, Fz, F3 & T3, F4, Fp2 & T3, F4, T5 \\
\hline V6 & Fpz, F4, F7 & Fpz, F8, F4 & Fp1, F4, T3 & Fp1, F4, T3 \\
\hline V7 & Fp2, Fpz, Fz & Fp2, T5, T6 & F7, T5, T3 & F7, T5, T3 \\
\hline V8 & Fpz, Fp2, F3 & Fpz, Fp2, F3 & Fp2, F7, T4 & Fz, T4, T5 \\
\hline V9 & T4, F3, Fp1 & T4, F3, T5 & T4, F3, F7 & T4, F3, F7 \\
\hline
\end{tabular}

After selecting the best three channels' combination as a result of the classification process, the result improvement analysis was performed with inter-label voting [37]. According to the decision mechanism, the result is explained for each epoch. This decision mechanism is summarized in Table 8. The label of two (or three channels) must be 1 to be considered the estimated epoch label 1 or "2D_3D”. On the other hand, if the estimated epoch label for two channels (or three channels) is 2 and considered “3D_2D”, it takes the value of 2 .

Thus, in the proposed hypothesis, in both feature extraction methods, only the 2, 3, and $4 \mathrm{~Hz}$ from the delta band and the temporal and frontal lobes from the EEG brain lobes were used to classify the 2D_3D and 3D_2D transitions. Meanwhile, the voting flow chart is displayed in Figure 5.

The 2D_3D and 3D_2D transitions' classification results for nine volunteers by using the voting process are presented in Table 9. In this table, the EEG signal analysis of each volunteer has been made for the channels of the volunteer that gives the best classification success. These channels were selected from the frontal and temporal lobes in all the volunteers, and this study is expected to be suitable for use in the biomedical field as a result of reducing the number of channels. Among the nine volunteers, the highest average test performance was calculated in V3 as $90 \%$ with SD, max feature extraction method, and LDA classifier. As seen in Table 7, this success was achieved by voting between channels Fz, T3, and T5. In general, based on Table 9, the best average test performance was determined as $76 \%$ in both classifications for both feature extraction methods. In addition, the classification performance criteria were largely compatible with each other.

Table 8 Decision mechanism

\begin{tabular}{|c|c|c|c|}
\hline Channel 1 & Channel 2 & Channel 3 & Estimated label \\
\hline $\mathbf{1}$ & $\mathbf{1}$ & $\mathbf{1}$ & $\mathbf{1}$ \\
\hline $\mathbf{1}$ & $\mathbf{1}$ & $\mathbf{2}$ & $\mathbf{1}$ \\
\hline $\mathbf{1}$ & 2 & $\mathbf{1}$ & $\mathbf{1}$ \\
\hline 1 & 2 & 2 & 2 \\
\hline 2 & 1 & 1 & 1 \\
\hline 2 & 1 & 2 & 2 \\
\hline 2 & 2 & 1 & 2 \\
\hline 2 & 2 & 2 & 2 \\
\hline
\end{tabular}




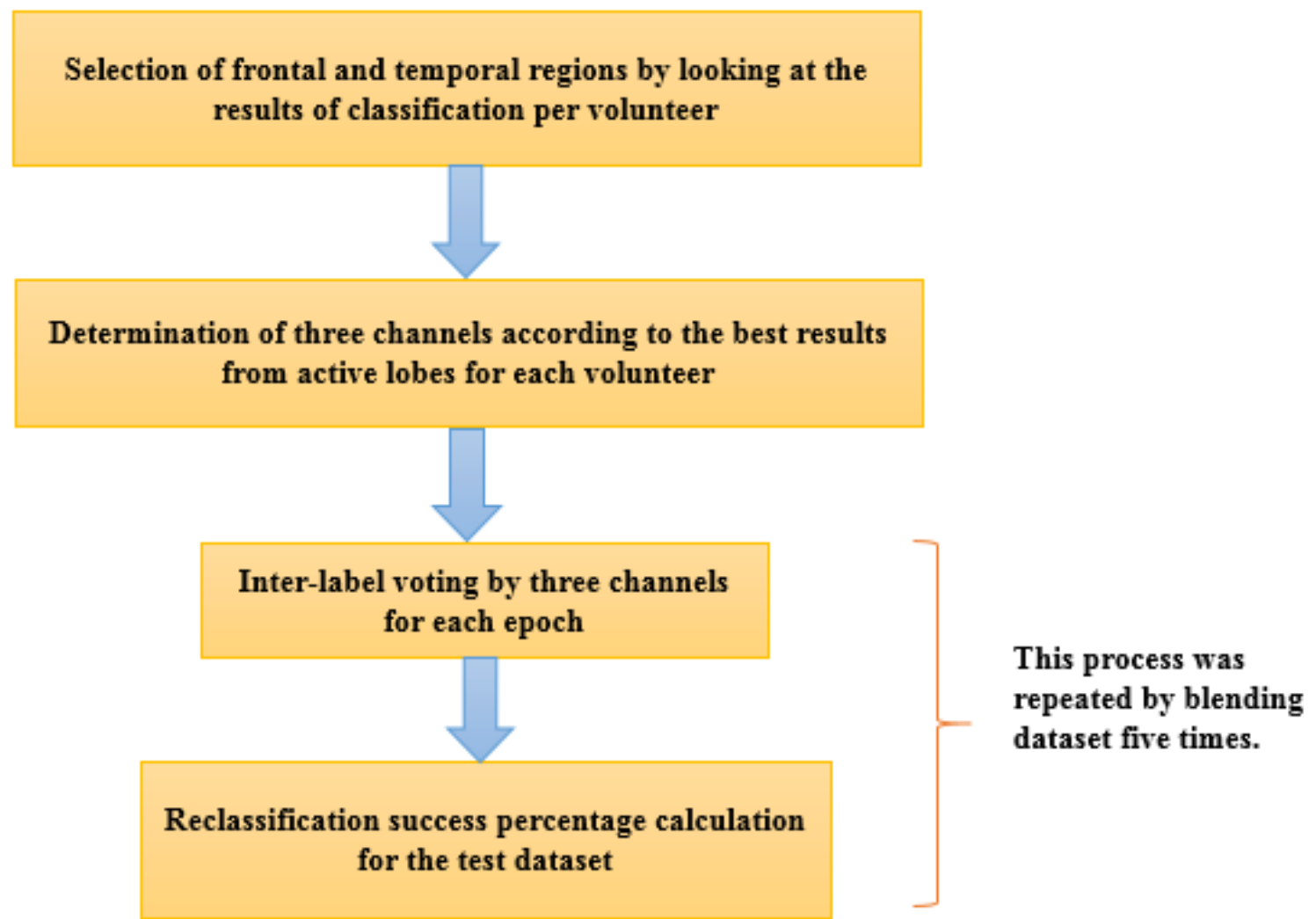

Figure 5 Voting flow chart of temporal and frontal lobe activity

\subsection{Classification Techniques and Performance Evaluation of Classification}

To achieve the goals of this study, namely 2D_3D and 3D_2D transition classification, k-NN and LDA classification techniques were employed. Since this study has two classes, the chance level is $50 \%$. Additionally, sensitivity and specificity [20] were used to evaluate classifier performance and classification accuracy. The basic definition of these techniques is briefly presented below.

The supervised and simple k-NN technique that is sensitive to the local structure of the data is also used in pattern recognition for different classification and regression processes [38]. This algorithm has a lazy learning mechanism, and it tries to make estimations by memorizing the training dataset instead of learning it. During the estimation process, it looks for the nearest neighbors, taking into account the entire data set [39]. The class of the new sample is determined by looking at the closest k neighbor's class according to the distance in the learning set. In this study, these distances were calculated using the Euclidean distance function. Setting the $\mathrm{k}$ or neighborhood parameter directly affects the performance of this algorithm. If $\mathrm{k}$ is too small, it affects noise sensitivity, and it is too large, it affects the neighbors of other classes. There is no clear method for choosing $\mathrm{k}$. As used in this study, in general, the optimum $\mathrm{k}$ is selected by using the cross-validation method.

The LDA algorithm is used in machine learning and pattern recognition to separate two or more classes of problems. Its basic principle is based on the linear combination of features. This algorithm actually reveals the modeling differences of classes by moving the high-dimensional space features to a smaller size [40].

To evaluate the classification result validation, K-fold cross-validation was applied. K-fold crossvalidation was employed to evaluate the classification result validation. In this study, the $\mathrm{K}$ value was chosen as 10. After cross-validation, the $\mathrm{k}$ value of $\mathrm{k}-\mathrm{NN}$ was obtained. Here, class 2D_3D and 3D_2D were defined as the positive and negative sample, respectively. To move on to the classification stage, the epochs of each class were randomly divided into two test and training sets. The classification process was repeated 20 times to avoid unlucky splitting. 
Manshouri et. al

Table 9 Results of classifying 2D_3D and 3D_2D transitions after the voting process in the temporal and frontal lobe activity

\begin{tabular}{|c|c|c|c|c|c|}
\hline & \multicolumn{2}{|c|}{ k-NN (\%Success) } & \multicolumn{2}{|c|}{ LDA (\%Success) } \\
\hline & & SD, max & Hjorth & SD, max & Hjorth \\
\hline \multirow{3}{*}{ V1 } & Accuracy & 63 & 69 & 60 & 85 \\
\hline & Specificity & 64.95 & 61.76 & 62.5 & 81.82 \\
\hline & Sensitivity & 62 & 100 & 58.33 & 88.89 \\
\hline \multirow{3}{*}{ V2 } & Accuracy & 66 & 68 & 80 & 80 \\
\hline & Specificity & 68 & 100 & 75 & 80 \\
\hline & Sensitivity & 68.02 & 61.34 & 88 & 80 \\
\hline \multirow{3}{*}{ V3 } & Accuracy & 63 & 67 & $\underline{90}$ & 65 \\
\hline & Specificity & 57.6 & 69.06 & 83.33 & 66.67 \\
\hline & Sensitivity & 100 & 66.1 & 100 & 63.64 \\
\hline \multirow{3}{*}{ V4 } & Accuracy & 78 & 79 & 75 & 75 \\
\hline & Specificity & 76.09 & 78.55 & 66.67 & 66.67 \\
\hline & Sensitivity & 80.61 & 79.56 & 100 & 100 \\
\hline \multirow{3}{*}{ V5 } & Accuracy & 74 & 79 & 85 & 85 \\
\hline & Specificity & 72.89 & 74.54 & 88.89 & 88.89 \\
\hline & Sensitivity & 79.38 & 88.42 & 81.82 & 81.82 \\
\hline \multirow{3}{*}{ V6 } & Accuracy & 80 & 80 & 75 & 75 \\
\hline & Specificity & 83.03 & 82.59 & 77.78 & 77.78 \\
\hline & Sensitivity & 78.56 & 78.78 & 72.73 & 72.73 \\
\hline \multirow{3}{*}{ V7 } & Accuracy & 76 & 74 & 75 & 75 \\
\hline & Specificity & 70.04 & 67.56 & 69.23 & 69.23 \\
\hline & Sensitivity & 91.11 & 92.7 & 85.71 & 85.71 \\
\hline \multirow{3}{*}{ V8 } & Accuracy & 76 & 73 & 65 & 65 \\
\hline & Specificity & 74.08 & 73.7 & 63.64 & 63.64 \\
\hline & Sensitivity & 80.71 & 74.63 & 66.67 & 66.67 \\
\hline \multirow{3}{*}{ V9 } & Accuracy & 82 & 80 & 80 & 80 \\
\hline & Specificity & 86.62 & 80.56 & 80 & 80 \\
\hline & Sensitivity & 81.78 & 82.43 & 80 & 80 \\
\hline \multirow{3}{*}{ Avearge } & Accuracy & 73 & 74 & $\underline{76}$ & $\underline{76}$ \\
\hline & Specificity & 72.58 & 76.48 & 74.11 & 80.44 \\
\hline & Sensitivity & 80.21 & 74.97 & 81.42 & 79.94 \\
\hline
\end{tabular}

It's good to say that in this study, classification performance was evaluated using confusion matrix. The general diagram of this matrix was shown in Table. 10. As an example, the confusion matrices for volunteer 1, 3, 5 and 9 using the Hjorth feature extraction method and LDA algorithm were presented in Figure 6. 
Table 10 Confusion matrix

\begin{tabular}{|c|l|c|c|}
\hline \multicolumn{2}{|l|}{ Volunteer } & \multicolumn{2}{c|}{ Predicted } \\
\cline { 3 - 4 } \multicolumn{2}{|c|}{ Observed } & 3D_2D & 2D_3D \\
\cline { 2 - 4 } & 2D_3D & TN & FP \\
\hline
\end{tabular}

\begin{tabular}{|c|c|c|c|}
\hline \multicolumn{2}{|c|}{ V1 } & \multicolumn{2}{c|}{ Predicted } \\
\cline { 3 - 4 } \multicolumn{2}{|c|}{ Observed } & 3D_2D & 2D 3D \\
\cline { 2 - 4 } & 2D_3D & 9 & 2 \\
\hline
\end{tabular}

\begin{tabular}{|c|c|c|c|}
\hline \multicolumn{2}{|c|}{ V3 } & \multicolumn{2}{c|}{ Predicted } \\
\cline { 3 - 4 } \multicolumn{2}{|c|}{ Observed } & 3D_2D & 2D 3D \\
\cline { 2 - 4 } & 2D_3D & 6 & 3 \\
\cline { 2 - 4 } & & 4 & 7 \\
\hline
\end{tabular}

\begin{tabular}{|c|c|c|c|}
\hline \multicolumn{2}{|c|}{ V5 } & \multicolumn{2}{c|}{ Predicted } \\
\cline { 3 - 4 } \multicolumn{2}{|c|}{} & 3D_2D & 2D_3D \\
\hline Observed & 3D_2D & 8 & 1 \\
\cline { 2 - 4 } & 2D_3D & 2 & 9 \\
\hline
\end{tabular}

\begin{tabular}{|l|l|c|c|}
\hline \multicolumn{2}{|c|}{ V9 } & \multicolumn{2}{c|}{ Predicted } \\
\cline { 3 - 4 } \multicolumn{2}{|c|}{} & 3D_2D & 2D_3D \\
\hline Observed & 3D_2D & 8 & 2 \\
\cline { 2 - 4 } & 2D_3D & 2 & 8 \\
\hline
\end{tabular}

Figure 6 The confusion matrices for volunteer 1, 3, 5, 9, using the Hjorth feature extraction method and in LDA algorithm

\section{Discussion}

In terms of the findings of the scenario, a comprehensive qualitative classification study was obtained after creating EEG records datasets in the analysis of 2D_3D and 3D_2D transitions. In a video consisting of single-stream 2D_3D and 3D_2D random transitions, the behavior of all brain lobes in the EEG frequency bands was studied in detail. In the literature review in the field of 2D and 3D technology, the analysis was started based on some basic information, e.g., by taking into account only the effectiveness of some brain lobes or frequency bands [41], [17], [9]. However, in the proposed study, the dominant EEG band and brain lobes [9] were selected by analyzing all the criteria.

In the literature review phase, no 2D_3D and 3D_2D transition classification hypothesis study was found to capture the moment of transition to sleep. Therefore, this study has great originality and innovation in the field of 2D and 3D technology. Studies in this field have been based on eye fatigue created by 3D televisions, superficial and qualitative brain signal analysis of $2 \mathrm{D}$ and 3D game consoles [2], [42], [43], [44]. Although it has been suggested that watching TV in 2D and 3D modes in terms of neurophysiological and cognitive responses does not have major effects on brain dynamics [2], it has been reported in later studies that this result changes in deep analysis [10], [45].

In the inter-volunteer classification results, the channels of the frontal and temporal lobes are the most effective channels in this transition classification analysis of the proposed study. Thus, the delta band and the channels of frontal and temporal lobes appear to be effective in the 2D_3D and 3D_2D transition classification. Based on the overall average results, the parietal lobe is considered to be important in the 2D_3D and 3D_2D transition classification. The presence of channels from the temporal lobe can be explained because of its important role in visual memory. In terms of the frontal lobe channels, this may be because the lobe has an important share of the focus of attention. The function of the parietal lobe is to interpret sensory information from different parts of the brain. Considering the PSD spectrogram graphics based on STFT, frequencies 2, 3, and $4 \mathrm{~Hz}$ from the delta band were chosen as the dominant band in these transitions. In this classification study, the importance of transition analysis is based on the eye structure. The human eye sees 3D due to its nature [20]. We proceed in this study, claiming that the ability to see in a sleepy and tired state will lose dimension. Therefore, the analysis and classification of 2D_3D and 3D_2D transition are the main objectives of research.

\section{Conclusion}


This paper investigated how watching a video consisting of random 2D and 3D parts affects the neural activity of the cortex. One of the innovations of this study was making 2D_3D and 3D_2D transition classification using a single-stream anaglyph video. For these transitions, especially considering the dominant time intervals, the brain activity for different frequency bands and brain lobes was considered. In this study, channels of five brain lobes and all frequency bands were tested in detail and dominant band and effective brain regions were obtained. This stage could be defined as EEG signal preliminary analysis and considered as an important principle of this study compared to other EEG studies. By interpreting the spectrogram charts, the PSD of the brain signals in the transitions was evident at the 2, 3 , and $4 \mathrm{~Hz}$ of the delta band. It was aimed to reach the target by extracting appropriate features to enhance the performance of classification algorithms from the selected band. The Hjorth parameters and the LDA classification technique yielded promising results in the temporal and frontal lobes in general.

The detailed analysis of the transition moment in a single-stream hybrid video consisting of 2D and 3D parts has the ability to add a new and unique perspective to driver fatigue studies. The results of this study provide a good basis for our future research. It is hoped that this study may shed light on the early diagnosis of the moment of falling asleep when it is conducted comprehensively on tired individuals. Using different feature extraction and classification techniques, the transition moment can be analyzed in professional videos. In addition to these planning, the number of participants can be increased to solve one of the limitations of the study. The classification results of 2D_3D and 3D_2D transition analysis can be improved using deep learning algorithms.

\section{References}

[1] R. N. H. R. Khairuddin, A. S. Malik, and N. Kamel, "EEG Topographical Maps Analysis for 2D and 3D Video Game Play," 2014 5th International Conference on Intelligent and Advanced Systems (ICIAS), no. June, pp. 1-4, 2014.

[2] H.-G. Jeong et al., "The impact of 3D and 2D TV watching on neurophysiological responses and cognitive functioning in adults," The European Journal of Public Health, vol. 25, no. 6, pp. 1047-1052, Dec. 2015.

[3] Q. Wang, O. Sourina, and M. K. Nguyen, "EEG-Based \&quot;Serious\&quot; Games Design for Medical Applications," in 2010 International Conference on Cyberworlds, 2010, pp. 270276.

[4] P. Benzie et al., “A Survey of 3DTV Displays: Techniques and Technologies,” IEEE Transactions on Circuits and Systems for Video Technology, vol. 17, no. 11, pp. 1647-1658, Nov. 2007.

[5] M. S. Banks, J. C. A. Read, R. S. Allison, and S. J. Watt, "Stereoscopy and the Human Visual System,” SMPTE Motion Imaging Journal, vol. 121, no. 4, pp. 24-43, May 2012.

[6] D. Bavelier, C. S. Green, D. H. Han, P. F. Renshaw, M. M. Merzenich, and D. A. Gentile, "Brains on video games," Nature Reviews Neuroscience, vol. 12, no. 12, pp. 763-768, Dec. 2011.

[7] M. Gentzkow, “Television and Voter Turnout*,” Quarterly Journal of Economics, vol. 121, no. 3, pp. 931-972, Aug. 2006.

[8] N. Manshouri, M. Maleki, and T. Kayikcioglu, "An EEG-based stereoscopic research of the PSD differences in pre and post 2D\&3D movies watching," Biomedical Signal Processing and 
Control, vol. 55, Jan. 2020.

[9] N. Zwezdochkina and V. Antipov, "The EEG Activity during Binocular Depth Perception of 2D Images,” Computational intelligence and neuroscience, vol. 2018, pp. 1-7, 2018.

[10] N. Manshouri, M. Maleki, and T. Kayıkçığlu, "Classification of Human Vision Discrepancy during Watching 2D and 3D Movies Based on EEG Signals," International Journal of Computer Science and Information Security, vol. 15, no. 2, pp. 430-436, 2017.

[11] D. De Waard, “The Measurement of Drivers’ Mental Workload,” 1996.

[12] B. J. Fisch and R. Spehlmann, Fisch and Spehlmann's EEG primer : basic principles of digital and analog EEG. Elsevier, 1999.

[13] V. Nityananda and J. C. A. Read, "Stereopsis in animals: evolution, function and mechanisms.,” The Journal of experimental biology, vol. 220, no. Pt 14, pp. 2502-2512, 2017.

[14] J. C. A. Read, "What is stereoscopic vision good for?,” 2015.

[15] M.-M. Hamed, E. Marzieh, and Ag. David, "The relationship between binocular vision symptoms and near point of convergence,” Indian Journal of Ophthalmology, vol. 61, no. 7, p. 325, Jul. 2013.

[16] C. WHEATSTONE, "On some remarkable and hitherto unobserved phenomena of binocular vision.,” The Optometric weekly, vol. 53, pp. 2311-5, Nov. 1962.

[17] M. Z. Ramadan et al., "Effects of Viewing Displays from Different Distances on Human Visual System,” Applied Sciences, vol. 7, no. 11, p. 1153, Nov. 2017.

[18] H. R. Khairuddin et al., "Analysis of EEG Signals Regularity in Adults during Video Game Play in 2D and 3D," 2013 35th Annual International Conference of the IEEE Engineering in Medicine and Biology Society (EMBC), pp. 2064-2067, 2013.

[19] Z. Minchev, "2D vs 3D Visualization and Social Networks Entertainment Games: A Human Factor Response Case Study,” Springer, Berlin, Heidelberg, 2013, pp. 107-113.

[20] N. Manshouri and T. Kayikcioglu, "A Comprehensive Analysis of 2D\&3D Video Watching of EEG Signals by Increasing PLSR and SVM Classification Results,” The Computer Journal, May 2019.

[21] W. Avarvand, Forooz Shahbazi and Bosse, Sebastian and Muller, Klaus-Robert and Schufer, Ralf and Nolte, Guido and Wiegand, Thomas and Curio, Gabriel and Samek, "Objective quality assessment of stereoscopic images with vertical disparity using EEG,” Journal of neural engineering, vol. 14, no. 4, pp. 1-14, 2017.

[22] S. Ting, T. Tan, G. West, A. Squelch, and J. Foster, "Quantitative assessment of 2D versus 3D visualisation modalities," in 2011 Visual Communications and Image Processing (VCIP), 2011, pp. 1-4. 
[23] A. Carvajal, "Quantitative Comparison between the Use of 3D vs 2D Visualization Tools to Present Building Design Proposals to Non-Spatial Skilled End Users,” in Ninth International Conference on Information Visualisation (IV’05), pp. 291-294.

[24] S. E. Kober, J. Kurzmann, and C. Neuper, "Cortical correlate of spatial presence in 2D and 3D interactive virtual reality: An EEG study,” International Journal of Psychophysiology, vol. 83, no. 3, pp. 365-374, Mar. 2012.

[25] Y. Han, H. Y. Lin, and C. Chen, "SP-3 Visual Fatigue for Laser-Projection Light-Field 3D Display in Contrast with 2D Display," 2017 24th International Workshop on Active-Matrix Flatpanel Displays and Devices (AM-FPD, pp. 9-12, 2017.

[26] F. P. S. Fischmeister and H. Bauer, "Neural correlates of monocular and binocular depth cues based on natural images : A LORETA analysis," Vision research, vol. 46, no. 20, pp. 33733380, 2006.

[27] (1) 3D Video Chain Saw! - YouTube. .

[28] “Xilisoft 3D Video Converter - 3D converter, convert to 3D video.” [Online]. Available: http://www.xilisoft.com/3d-video-converter.html. [Accessed: 30-Dec-2019].

[29] "Download Free 3D Video Converter - Convert 2D to 3D | IQmango Free Software.” [Online]. Available: http://iqmango.com/3DVideo_Converter.html. [Accessed: 30-Dec-2019].

[30] “Easiest Video Editing Software Free Download.” [Online]. Available: http://www.idooeditor.com/. [Accessed: 30-Dec-2019].

[31] J. Kim et al., "A full-color anaglyph three-dimensional display system using active color filter glasses,” Journal of Information Display, vol. 12, no. 1, pp. 37-41, Mar. 2011.

[32] K. Eroğlu, T. Kayıkçığlu, and O. Osman, "Effect of brightness of visual stimuli on EEG signals,” Behavioural Brain Research, vol. 382, p. 112486, Mar. 2020.

[33] N. Roehri, J. M. Lina, J. C. Mosher, F. Bartolomei, and C. G. Benar, "Time-Frequency Strategies for Increasing High-Frequency Oscillation Detectability in Intracerebral EEG,” IEEE Transactions on Biomedical Engineering, vol. 63, no. 12, pp. 2595-2606, Dec. 2016.

[34] N. Kehtarnavaz, "Frequency Domain Processing,” in Digital Signal Processing System Design, Elsevier, 2008, pp. 175-196.

[35] F. Hlawatsch and G. F. Boudreaux-Bartels, "Linear and quadratic time-frequency signal representations,” IEEE Signal Processing Magazine, vol. 9, no. 2, pp. 21-67, Apr. 1992.

[36] S.-H. Oh, Y.-R. Lee, and H.-N. Kim, “A Novel EEG Feature Extraction Method Using Hjorth Parameter."

[37] T. Kayikcioglu, M. Maleki, and K. Eroglu, "Fast and accurate PLS-based classification of EEG sleep using single channel data,” Expert Systems with Applications, vol. 42, no. 21, pp. 7825- 
7830, Jun. 2015.

[38] E. Fix and J. L. Hodges, "Discriminatory Analysis. Nonparametric Discrimination: Consistency Properties,” International Statistical Review / Revue Internationale de Statistique, vol. 57, no. 3, p. 238, Dec. 1989.

[39] N. S. Altman, “An introduction to kernel and nearest-neighbor nonparametric regression,” American Statistician, vol. 46, no. 3, pp. 175-185, 1992.

[40] A. M. Martinez and A. C. Kak, "PCA versus LDA," IEEE Transactions on Pattern Analysis and Machine Intelligence, vol. 23, no. 2, pp. 228-233, Feb. 2001.

[41] M. Host'ovecký and B. B, "Brain activity: beta wave analysis of 2D and 3D serious games using EEG,” JAMSI, vol. 13, no. 2, 2017.

[42] C. Chen, K. Li, Q. Wu, H. Wang, Z. Qian, and G. Sudlow, "EEG-based detection and evaluation of fatigue caused by watching 3DTV,” Displays, vol. 34, no. 2, pp. 81-88, Apr. 2013.

[43] C. Chen et al., "Assessment visual fatigue of watching 3DTV using EEG power spectral parameters,” Displays, vol. 35, no. 5, pp. 266-272, Dec. 2014.

[44] A. S. Malik et al., "EEG based evaluation of stereoscopic 3D displays for viewer discomfort," BioMedical Engineering OnLine, vol. 14, no. 1, p. 21, Dec. 2015.

[45] S. Kim and D. Kim, "Differences in the Brain Waves of 3D and 2 . 5D Motion Picture Viewers," arXiv preprint arXiv:1210.2147. 